TM-1411

0102.000

(SSC-N-236)

\title{
Passive Correction of Persistent Current Multipoles in Superconducting Accelerator Dipoles*
}

H.E. Fisk, R.A. Hanft, M. Kuchnir, and A.D. McInturff

July 1986

*Submitted to the ICFA Workshop on Superconducting Magnets and Cryogenics, Brookhaven National Laboratory, Upton, New York, May 12-16, 1986 
H. E. Elsk, R. A. Hanft, M. Kuchnir, and A. D. MoInturff Ferml National Accelerator Laboratory

P. 0. Box 500

Batavia, IL 60510

Abstract. - Correction of the magnetization sextupole and decapole fields with strips of superconductor placed just inside the coll winding is discussed. Calculations have been carried out for such a scheme, and tests have been conducted on a $4 \mathrm{~cm}$ aperture magnet. The calculated sextupole correction at the injection excitation of $330 \mathrm{~A}, 5 \%$ of full fleld, was expected to be $77 \%$ effectlve, while the measured correction is $83 \%$. thus suggesting the gcheme may be useful for future accelerators such as SSC and LHC.

\section{INTRODUCTION}

The persistent currents in superconducting fllaments generate unwanted multipole fields in accelerator magnets. These fields, typlcally a few gauss at the reference radlus, present a challenge to the accelerator builder since such flelds must be corrected if the accelerator is to operate over a large range, such as injection at $5 \%$ of the top energy. These fields may be corrected with full length correction colls inside the dlpoles, or it may be possible, with very small diameter fllaments, to correct these hysteretic fields with correction elements located at one place in each half cell of the lattice. Full length correction colls that are driven with external power supplies or that are shorted and driven by unwanted flux have been discussed, ${ }^{1,2}$ The device described here is different in that no power supply is required and no coil of exacting dimensions is used.

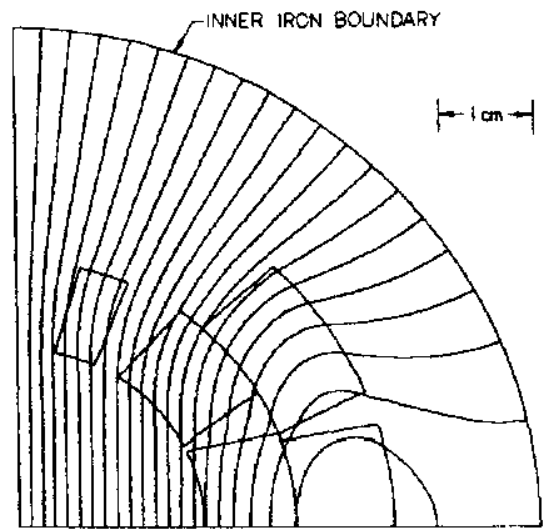

Figure 1. Sketch of the first quadrant of the SSC design $D 4 \mathrm{~cm}$ aperture dipole cross section showing conductor blocks and magnetic field lines.

* Work supported by the U. S. Department of Energy
The theory of induced magnetization by persistent currents is discussed in papers by C. P. Bean ${ }^{3}$ and M. A. Green." The positive and negative current density induced in a single filarment, due to the flux change caused by ramping the magnet, creates a source of magnet1c dipoles that persist as long as the magnet is superconducting. Because of the coll winding geometry, these persistent current dipoles generate geometrically allowed macroscoplc flelds: dipole, sextupole, decapole, etc. Brown and Fisk have described a way to harmonlcally correct the persistent current fields with longitudinal strips of superconductor located inside the coil where harmonically the field is primarlly dipole. ${ }^{5}$ This paper describes a test of a $4 \mathrm{~cm}$ aperture 1 meter long SSC design D dipole outfitted with a corrector that employs 32 strips of approximately $1.2 \mathrm{~mm}$ square cross section copper and superconductor.

\section{PRE-CORRECTION MAGNET TEST}

A sketch of the first quadrant of the dipole winding cross section and its magnetic fleld are shown in Figure 1. The two layer coll design has 2 (1) wedges in the inner (outer) layer to improve field quality. To calculate the persistent current sextupole the induced magnetization of the cable must be known as well as the local fleld Inside the winding. Figure 2 shows cable magnetization as a function of external magnetic field for the inner layer design $D$ cable that is made from $23 \mathrm{strands}$ of $.808 \mathrm{~mm}$ wire with $23.8 \mu \mathrm{m}$ fllaments and the outer cable that is made from 30 strands of .648 mo wire with $16.4 \mu \mathrm{m}$ filaments.

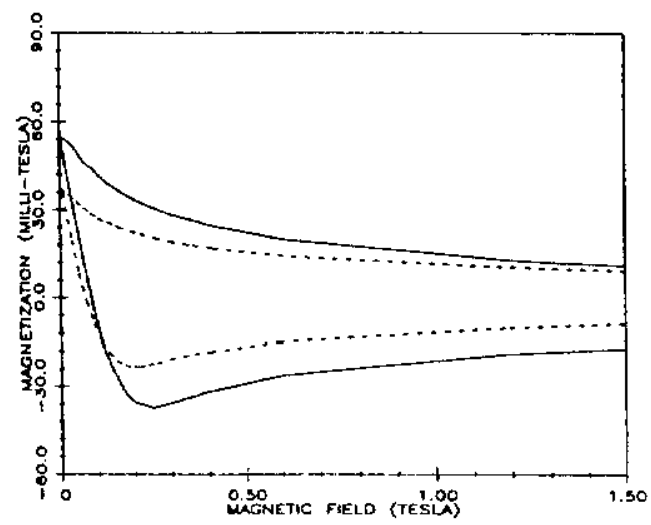

Figure 2. Measured cable magnetization. The solid (dashed) line refers to cable used in the inner (outer) layer. 
The multipole expansion for the principal component. $B_{y}$, of the magnetic field in polar coordinates $(r, \theta)$ is

$$
\begin{aligned}
& B_{y}=\bar{B} \sum_{n=0}^{\infty}\left(\frac{r}{p}\right)^{n}\left(b_{n} \cos n \theta-a_{n} \sin n \theta\right) \\
& b_{a}=1.0 \\
& a_{0}=0.0
\end{aligned}
$$

where $\bar{B}$ is the magnitude of the dipole field; $p$ is the reference radius, here equal to $1 \mathrm{~cm}$; and $b_{n}$

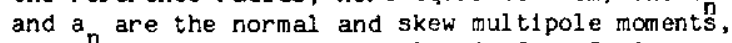
respectively. The pole number is $2 n+2,1 . e$. the normal sextupole moment is $b_{2}$. It is customary to report $b_{n}$ and $a_{n}$ in "units" obtained by suppressing a factor of $10^{-4}$. The multipole flelds $B^{\prime}$ due to persistent currents can be calculated from an integral over the coll cross section:

$$
B_{n}^{\prime}=\vec{B} b_{n}^{\prime}=\frac{3(n+1) \mu_{0}}{4 B} \int \frac{M(B) \cos \left[(n+2) \theta_{c}-\alpha\right] d A}{r_{c}^{n+2}} ;
$$

where the magnetization $M(B)$ is obtained by direct measurement. Here $\left(r, \theta_{\rho}\right)$ are polar coordinates for the point of integration in the winding, dA = $r d r d \theta, B$ is the ratio of the total coll area to metaf area, and the angle $\alpha$ is the direction of the induced dipoles relative to the main dipole direction. The moments b' add to the moments generated by the excitation current to give the total monents $b_{n}$.

The theory cited above relates $M$ to other cable properties by

$$
M=\frac{4}{3 \pi} \frac{\varepsilon J_{C} D}{1+C / S} .
$$

Here $\varepsilon$ is the penetration factor, $J$ is the critical current density, and $D$ the filament diameter. The quantity $\mathrm{C} / \mathrm{S}$ is the area ratio of copper to superconductor. Fl gure 3 shows the calculated contributions to the sextupole field for inner and outer layers as a function of $\theta$ at injection, where the central dipole field is ăbout $0.3 \mathrm{~T}$.

Multipole data are obtained from voltage Induced in a Morgan coll rotating at $6 \mathrm{~Hz}$ inside a warm finger located in the cold magnet bore. The voltages are transferred via slip-rings to el ther a BNL style magnetometer' or a lock-in amplifier measuring system. For the magnetometer analysis, square wave gates are generated that can be used to multiply the Morgan coil induced signals which are then sampled and digitized. The measured dipole field is obtalned by gating the dipole Morgan coil with a synchronous signal that is positive for the first half cycle of rotation and negative for the second hal $f ; 1 . e$. a $6 \mathrm{~Hz}$ square wave ( 10 gate). Sextupole fields are obtained with an $18 \mathrm{~Hz}$ square wave ( 30 gate) applied to the output of the sextupole Morgan coll. Other multipoles such as quadrupole, octupole, decapole, etc, are obtained by using the proper gate/coil

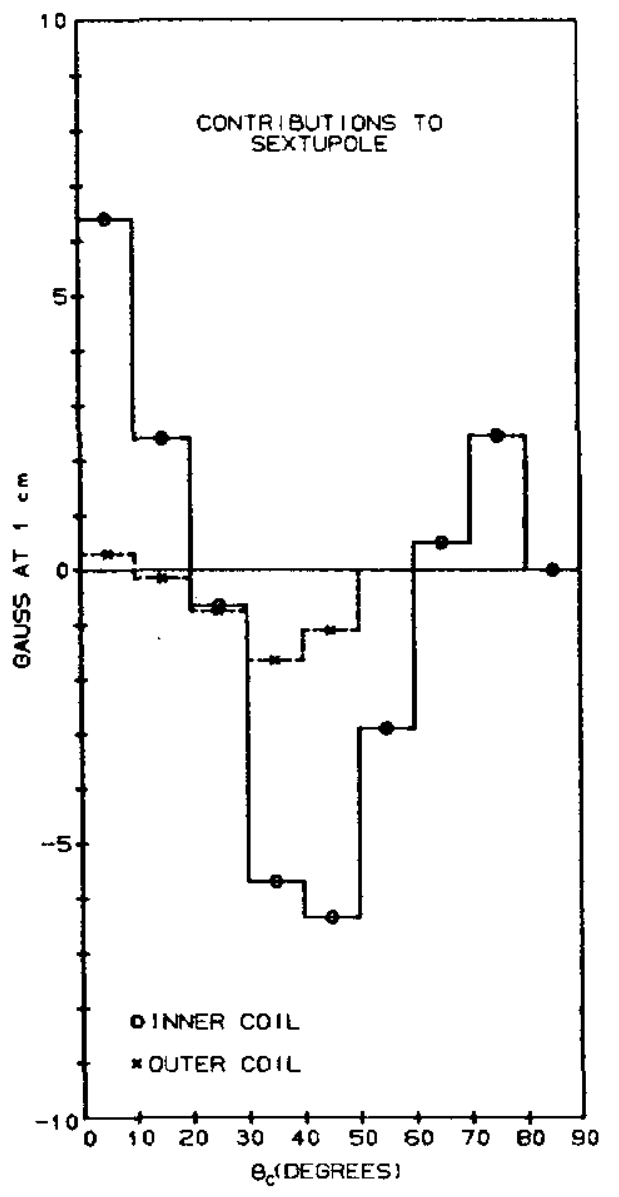

Figure 3. Angular dependence of the persistent current contributions to the sextupole field for both the inner and outer layers of the coll.

combination. Both normal and skew maments can be determined by use of orthogonal square wave (1.e. the sine-like and cosine-like) gates. The lock-in amplffier measuring technique is similar except that the square wave gating signals serve as references to the lock-1n. Both measuring systems work well and results that are

independently processed agree. The magnetoneter is faster in that readout may be obtalned as frequently as every 0.5 seconds. For very small signals, especially those that result from low current in the magnet, the lock-in system is more accurate.

Figure 4 shows the normal measured sextupole moment of the magnet without corrector as a function of excitation current and the expected behavior calculated using the magnetization data shown in Figure 2. The data were obtalned for two different ramp rates, $2 \mathrm{~A} / \mathrm{s}$ and $12 \mathrm{~A} / \mathrm{s}$, with the magnetometer system. Although the agreement between the measured data and theory shows some 
systematio differences, the agreement at the injection current of $330 \mathrm{~A}$ is quite good. The only known effect not taken into account is the distortion that transport current may impose on the persistent currents. This effect is expected to be only a few percent at the fleld levels encountered near injection.

At $330 \mathrm{~A}$ the sextupole is 26 units below the value of -21 units due to transport current only. This b1g persistent current sextupole moment is due to the large diameter of the fllaments used in this early SSC model magnet. Smaller filaments. somewhere between 2 and $8 \mu \mathrm{m}$; are anticlpated for the final SSC magnets.

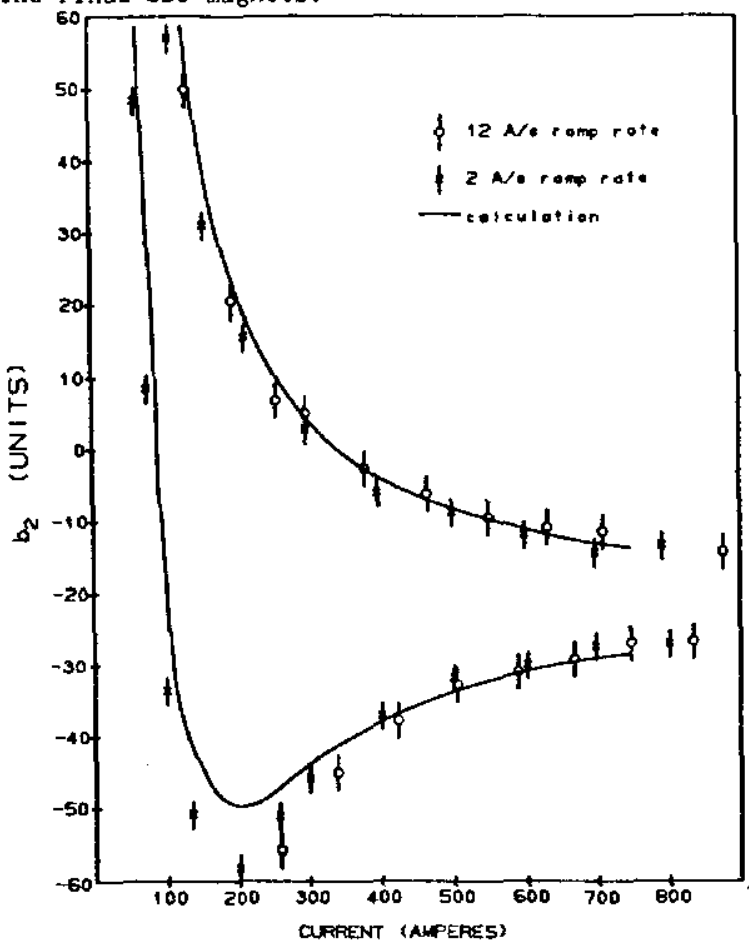

Figure 4. Measured and predicted normal sextupole moment for the uncorrected magnet vs. excitation current. Data were taken with ramp rates of $2 A / S$ and $12 \mathrm{~A} / \mathrm{s}$.

\section{PASSIVE CORRECTOR}

A photograph of the pasalve corrector is shown in Figure 5 along with the magnet cross section. The $4 \theta$ placement of the passive strips is designed to correct the sextupole moment $b_{2}$ at low fields without blasing $b_{2}$ at hf $h$ h field.

Rectangular wire with $45 \mu \mathrm{m}$ filaments was readily available for use in the passive corrector. If time and cost were not factors, the ideal choice of corrector filament diameter would probably fall in the $30 \mu \mathrm{m}$ region since the full penetration of fllaments occurs over a smaller range of excitation current. More plainly, the corrector would be more effective at lower magnet excitation.

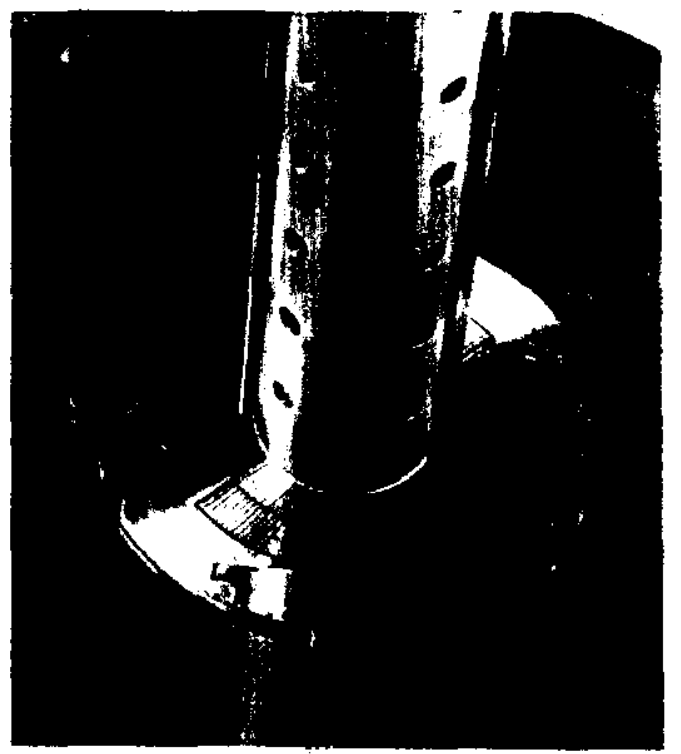

Figure 5. Photograph of the passive corrector and the design $D$ coil cross section.

The corrector, diagramatically shown in F1gure 6 , was made by machining 32 grooves in an al uninum pipe of inner and outer diameters 1.375" and $1.555^{\circ}$, respectively. The grooves were :054" wide by .051" deep such that the rectangular wires could be pressed into ther. The strips of passive corrector occupy the region $\pm 22.50^{\circ}$ about the $x$ and $y$ axes. The corrector wires are closely packed leaving about $0.022^{\prime \prime}$ of aluminum between each strip. There is no insulation on the superconducting wire and the aluminum pipe is perforated with holes to allow 11 quid he to freely circulate. The aluminum tube with corrector wire is azimuthally positioned by use of guide keys that fit into machined grooves at the top and bottom of the corrector. There are mating guide slots in the stainless steel collar poles that engage the guide keys. The corrector assembly is estimated to be positioned correctly in angle to about $\pm 7 \mathrm{mr}$.

The dimensions of the aluminum tube were chosen to allow the insertion of the multipole measuring apparatus including its warm finger. The al uninum tube 1 tself was not intended to simulate the accelerator beam tube, but was merely a convenient cholce from the machining point of view.

The corrector outside diameter is nominally $0.010 "$ smaller than the inside diameter of the coil. This space is filled with kapton insulation wrapped around the corrector. After the $1 \mathrm{~m}$ magnet DA1002 had its field measured without correction the corrector was inserted. This turned out to be a fairly elaborate procedure because the magnet was wound on the expanding mandrel at Fermilab and collared without using epoxy. Insertion of the corrector required the collars to be unclamped and in the process the 
placement of the conductors in the winding changed enough to give a slightly different transport current sextupole woment.

\section{TEST RESULTS AND PREDICTIONS}

Figure 7 shows the sextupole moment of DA1002 with the corrector installed as a function of current for three different ramp rates: $12 \mathrm{~A} / \mathrm{s}$, $6 \mathrm{~A} / \mathrm{s}$, and $\mathrm{dc}$. Here do means the excltation current was held constant 3 ong enough for the lock-in system to report a stable signal, typlcally 30 seconds. The data show a demonstrated ramp rate dependence for the sextupole field. This may be due to eddy currents induced in the aluminum pipe that supports the corrector strips. The proposed SSC ramp rate is $6 \mathrm{~A} / \mathrm{s}$ so that data may be compared oirectly ta the dc data to estimate what improvement would be gained with no ramp rate effects.

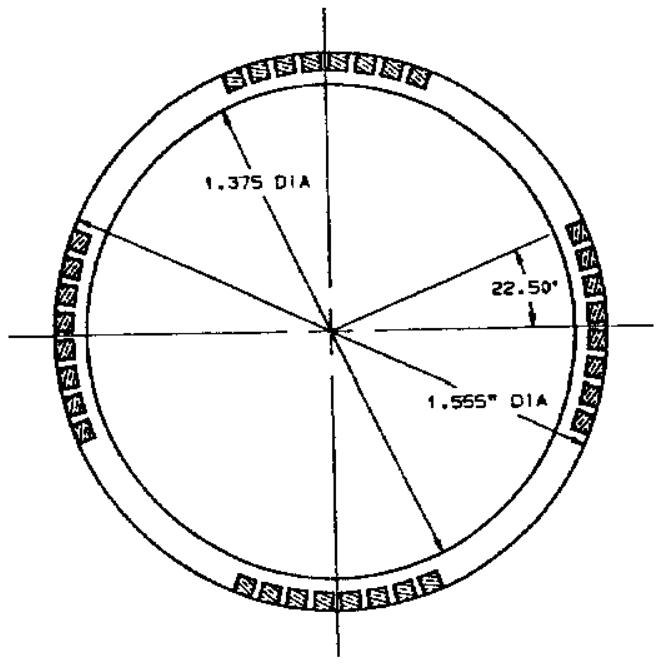

Figure 6. Dlagram of the locations of the passive corrector strips as press fitted to the aluminum pipe.

The measured sextupole moment for the dc case and two predictions are shown in Figure 8 along with the uncorrected magnet data. Both predictions use the magnetization for the corrector strips that is shown in Figure 9.6 In F1 gure 8 the curve labeled (a) uses the measured hysteresis sextupole fleld for the magnet alone plus the predicted corrector sextupole fleld while curve (b) results from adding the calculated sextupole for both the magnet coll and the corrector. Curve (a) is expected ta agree with the data better than (b) since less prediction is required. This seems to be the case although there is agaln a systematic difference between the prediction and observation. If one assumes curves (a) or (b) the antlolpated reduction in the sextupole moment at $330 \mathrm{~A}$ is in the range $67 \%$ to 77\%. The measured result is a reduction of $83 \%$. Although a slightly better result could be obtained with a different passive conductor arrangement, this is already a blg improvement over the uncompensated field and adequately demonstrates the passive corrector principle.

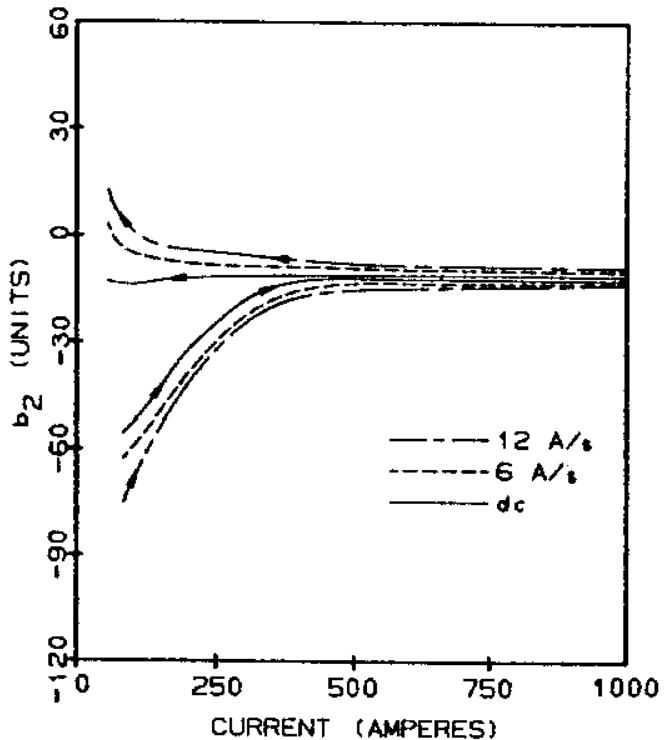

Figure 7. Measured sextupole moment for the passively corrected dipole DA1002. Three sets of data are shown: $12 \mathrm{~A} / \mathrm{S}$, $6 \mathrm{~A} / \mathrm{s}$ and $\mathrm{dc}$.

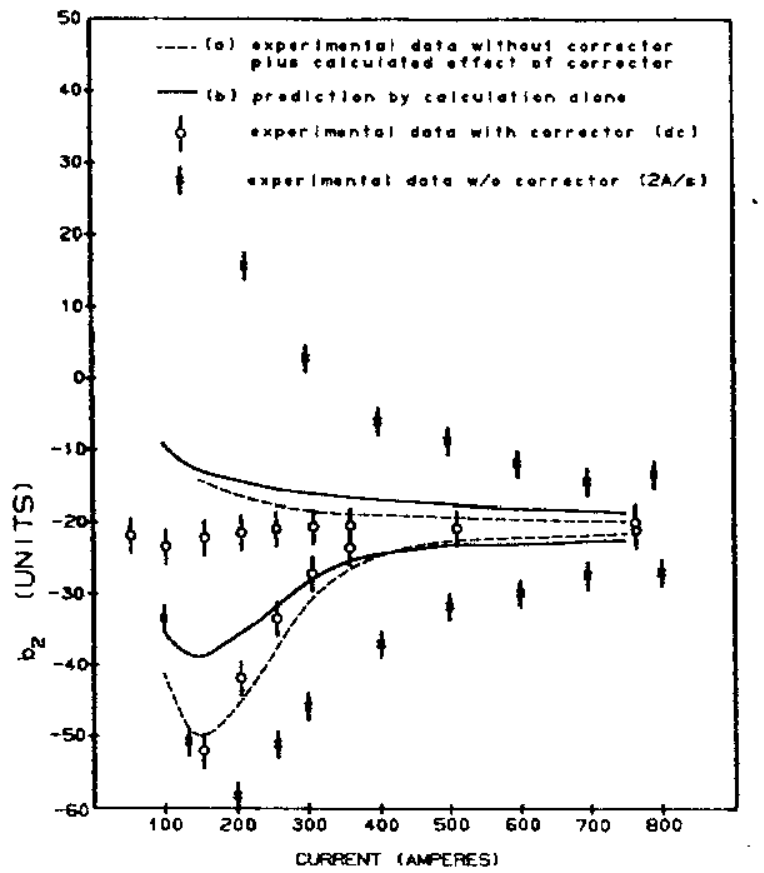

Figure 3. Comparison of the measured and predicted sextupole field. See the text for an explanation of the predictions. 
Although it is possible to build a corrector that is effective in simuitaneously correcting both sextupale and decapole hysteresis, the corrector described here is expected to have no effect on the decapole, and within errors the measurements so confirm.

$\cos T$

The monetary cost of making a correction this way $1 \mathrm{~s}$ of interest. For the example discussed here the NbTi in the magnet cross section is 148 $m^{2}$ per quadrant while the passive corrector's NbT1 is $6.1 \mathrm{~mm}^{2}$. Thus, the corrector superconductor cost is about 4.15 of the magnet superconductor. Given that the conductor for the dipoles will cost $\$ 300 \mathrm{M}$ the corrector superconduct or cost would be in the nelghborhood of $\$ 12$ to $\$ 15 \mathrm{M}$ if the area ratios could be kept to the 4 to 51 range. The cost of attaching the passive corrector to the beam tube is probably not more expensive than the cost of attaching the present multiwires and the placement is probably not as critical. There would also be cost savings relative to power supplies, cables, control circuitry and driving software.

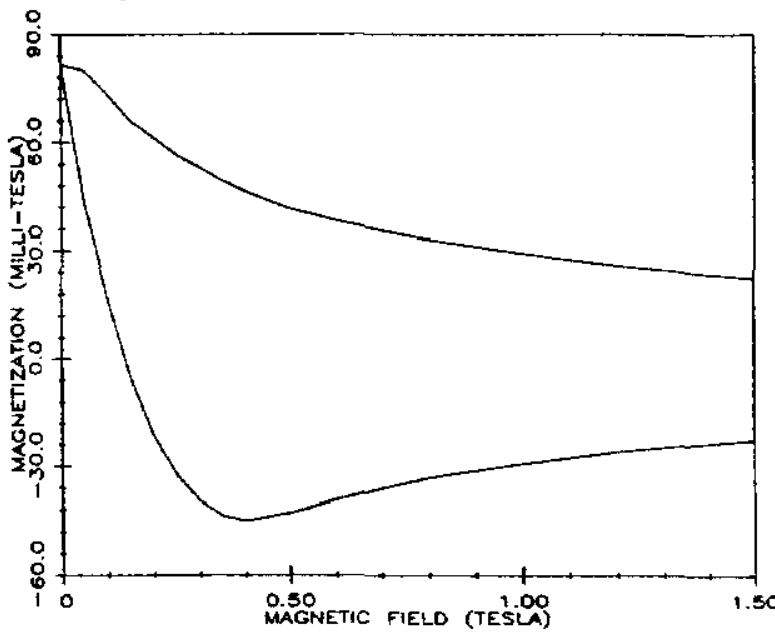

F1gure 9. Measured magnetization for the rectangular cross section wire used in the passive corrector.

\section{DISCUSSION}

If the field quality needed at injection can be obtained with this passive corrector there are a number of advantages over full length driven correction coils:

(1) automatic temperature compensation;

(2) elimination of power supplies, cables, electronics, etc.;

(3) no incorrect polarities (the fields can be reset by golng through a $\Delta I=500 \mathrm{~A}$ hysteresis loop);

(4) there is no transport current in the corrector so that in the event of a magnet quench the corrector cannot burn up;
(5) machine operators can't get confused - or at least they can't forget to turn on/off or send wrong current settings to the corrector

(6) the decapole field can be corrected simultaneously with the sextupoie field (additional superconductior would be needed);

(7) cost might be reduced signiflcantly.

There are also some disadvantages:

(1) no tuning is possible;

(2) lumped correctors are st11l required;

(3) there will be slightly higher superconductor hysteresis losses (a few percent);

(4) a slightly greater amount of radial space may be required for the corrector wire arrangement.

\section{FUTURE PROGRAM}

There are still a few tests to do with the present model, DA1002 and its compensator. The temperature independence must be checked and ramp rate dependence needs further investigation. If a new magnet is available a similar corrector could be fabricated on a different support plpe, e.g. G-10 or stainless steel.

When there is new small filament conductor avallable there will need to be new calculations and new rectangular wire with proper fllament diameter will need to be procured. This future test should include an attempt to null the decapole as well as the sextupole.

\section{REFERENCES}

[1] P. Thompson et al., Proceedings of this conference.

[2] w. Gilbert et al., IEEE Trans, on Magnetics MAG-21, $486(1985)$.

[3] C. P. Bean, Phys. Rev. Lett. 8, 250 (1962).

[4] M. A. Green, Residual Flelds in Superconducting Magnets, Proc. of the Magnet Technology Conf. MT-4, p 339 (1972)

Brookhayen National Laboratory.

[5] B. C. Brown and H. E. Fisk; A Technique to Minimize Persistent Current Muttipoles in Superconducting Accelerator Magnets; Proc. of the 1984 Summer Study on the Deslgn and Utilization of the Superconducting Super Collider p 336 (1985) Ed. R. Donaldson and J. G. Morfin.

[6] A. K. Ghosh, Brookhaven National Laboratory has graciously supplied all the measured magnetization data discussed in thls paper. See A. K. Ghosh and W. B. Sampson, Advance in Cryogenic Engineering 32, paper DZ-7 (1986).

[7] C. H. Holbrow, Isabelie Project Technical Note 170, Brookhaven National Laboratory (1979). 